\title{
Performance of a Metamaterial-based $1 \times 2$ Microstrip Patch Antenna Array for Wireless Communications Examined by Characteristic Mode Analysis
}

\author{
Ehab K. I. HAMAD ${ }^{1}$, Ahmed ABDELAZIZ ${ }^{2}$ \\ ${ }^{1}$ Dept. of Electrical Engineering, Faculty of Engineering, Aswan University, Aswan 81542, Egypt \\ ${ }^{2}$ Dept. of Electronics \& Comm. Engineering, Higher Institute of Engineering \& Technology, Luxor 85834, Egypt \\ e.hamad@aswu.edu.eg, ahmedabdalaziz15@yahoo.com
}

Submitted February 17, 2019 / Accepted September 9, 2019

\begin{abstract}
The theory of characteristic modes (TCM) is used to examine the behavior of hexagonal split ring resonator (HSRR) unit cells employed in the ground plane of a 2-element microstrip antenna array. Suppression of higher harmonics and reduction in mutual coupling between the elements as a result of metamaterial loading was investigated using TCM. The novelty of this paper is the use of the TCM to investigate the behavior of the HSRR, to reduce this mutual coupling, to significantly enhance the antenna's performance. The TCM is employed to precisely determine where the HSRR unit cells should be allocated to efficiently block the coupling modes and not affect the noncoupling modes. The simulation results showed that gains of $5.36 \mathrm{~dB}$ and $8.2 \mathrm{~dB}$ as well as bandwidths of $628 \mathrm{MHz}$ and $610 \mathrm{MHz}$ are achieved for the single and 2-element array antennas, respectively. The bandwidth of the array antenna was enlarged to $906 \mathrm{MHz}$ by loading the ground plane with five HSRR cells. Prototypes for the proposed antennas were fabricated and the experimental outcomes showed good agreement between the measurements and simulation results. The gain and radiation efficiencies were measured using the SATIMO Starlab anechoic chamber.
\end{abstract}

\section{Keywords}

Array antennas, characteristic mode analysis, metamaterial, microstrip patch antenna, theory of characteristic mode, wireless communications

\section{Introduction}

The mobile and wireless communications industry has seen rapid expansion in the recent decade, progressing from an analog platform to digital systems like $2 \mathrm{G}$ (GSM), and then advancing to high data rate wireless communication in WCDMA, also known as 3G. Evolution of the communication did not stop there, but kept its pace of development to $3.5 \mathrm{G}$ (HSPA) and then to $4 \mathrm{G}$, also known as LTE and LTE-A. Today, 5G pilot studies are in progress and the platform is predicted for release in the 2020s [1],
[2]. 5G technology that embraces the millimeter wave is required to overcome existing constraints such as slow data transfer rate and spectrum scarcity. The fifth-generation technology also offers better coverage as compared to that of the previous generations [3], [4]. In this regard, the microstrip antenna plays a vital role in this fast-growing industry today. Indeed, we can hardly think of any development in wireless communication without engaging microstrip antenna technology. To establish communication between wireless devices on higher frequency bands such as the mm-wave band, we need antennas which are conformal, small and compact, and that are cheap and easily fabricated. In this regard, printed patch antennas have been the preferred choice over other types of antennas.

The main drawbacks of microstrip patch antenna (MPA) are its lower gain and bandwidth. These features are being extensively improved upon through several approaches [5], some of which are: $(i)$ use of large number of patch antennas in array configuration [6]; (ii) increase of substrate thickness [7]; (iii) optimization of the impedance matching by using short pins, plates, posts, etc. [8]; (iv) use of high permittivity substrates [9]; $(v)$ modification of the basic patch shape [10]; (vi) use of multiple layer of substrates [11]; and (vii) for instance by the incorporation of metamaterials (MTM) [12].

Metamaterials are artificial materials that can be adapted to display specific electromagnetic properties not commonly found in nature. The theoretical concept of MTM was introduced by Veselago in 1968 [13]. The material has unique properties such as negative values of permittivity and permeability, negative refractive index, backward wave propagation, etc. [14]. Among its many applications, MTM is employed extensively to ameliorate various constraints in the antenna's performance and characteristics.

Authors in [15] designed a dual-band microstrip antenna incorporating a multiple split ring resonator (MSRR) to achieve size reduction. Saravanan et al. in [16] covered a conventional patch antenna with MTM superstrate for gain enhancement. More recently, Khandelwal et al. in [17] 
proposed a novel metamaterial structure showing negative capabilities at dual frequency bands and with a very small frequency ratio. Ali et al. in [18] designed a multiple circular and rectangular complementary split ring resonator (CSRR) to generate multiband response. Further recent progresses in this area have since been reported [19], [20].

In an antenna array, the mutual coupling effect typically deteriorates the radiation properties of the array. To avoid mutual coupling and grating lobes, separation between the elements should be between $\lambda_{\mathrm{g}} / 2$ and $\lambda_{\mathrm{g}}$, depending on the angular scan range [21]. Various methods have been assessed for the suppression of mutual coupling between the elements, including the neutralization technique [22], use of electromagnetic band gap (EBG) structures [23] or etching of slots or slits from the ground and forming defected ground structures (DGSs) [24]. Based on the TCM, we used MTM unit cells and characteristic mode analysis to reduce the mutual coupling between the two elements of the array antenna.

Characteristic mode analysis (CMA) has become a favorable tool for analyzing and designing antennas, mainly due to the physical insight gained about antenna operating principles without considering any particular feeding. Characteristic mode theory is a modal analysis technique for antennas of arbitrary shape. It also gives a clear understanding of the resonating frequency of specific modes, radiation patterns and the corresponding mode current. Developed by [25], TCM was subsequently modified by diagonalizing the impedance matrix [26]. Characteristic modes for antenna design provide information such as precise resonant frequencies and the right feed points to excite a particular CM. The detailed derivation of TCM as well as its application in antenna design may be found in [27]. The CMs can be obtained by using a generalized eigenvalue equation in the form of

$$
X\left(\mathbf{J}_{n}\right)=\lambda_{n} R\left(\mathbf{J}_{n}\right)
$$

where $X$ and $R$ represent the reactance and resistance, respectively of the geometry under consideration, $\lambda_{n}$ is an eigenvalue corresponding to eigenvector $\mathbf{J}_{n}$ which represents the current density of the $n^{\text {th }}$ mode on the antenna surface.

Another noteworthy parameter is the modal significance (MS), which is a parameter used to find the resonant frequency as well as the bandwidth (BW) offered by a mode. It is calculated as follows [25]:

$$
M S_{n}=\left|1 /\left(1+\mathrm{j} \lambda_{n}\right)\right| \text {. }
$$

The same information can be extracted from the characteristic angle curves given by

$$
\beta_{n}=180^{\circ}-\tan ^{-1}\left(\lambda_{n}\right)
$$

When a $\mathrm{CM}$ resonates, it is observed that $\lambda_{n}=0$ while $M S_{n}=1$ and $\beta_{n}=180^{\circ}$.

The CST Microwave Studio is used in this paper to apply the CMA. In CST MWS that we are using, a CMA of
MTM-based antennas and possibly of other structures is carried out with the multilayer solver available in the CST package. After applying the CMA to the structure, the results give the eigenvalue $\left(\lambda_{n}\right)$, characteristic angle $\left(\beta_{n}\right)$, and modal significance $\left(M S_{n}\right)$ parameters over the desired frequency band. From these three parameters, one can explore the behavior of the concerned structure. When the characteristic mode resonates, the value for $\lambda_{n}$ is equal to 0 while the $M S_{n}$ is equal to 1 and the value of $\beta_{n}$ is equal to $180^{\circ}$. When $\beta_{n}<180^{\circ}$ or when $\lambda_{n}<0$, this means that the mode stores magnetic energy, in otherwise when $\beta_{n}>180^{\circ}$ or when $\lambda_{n}>0$ it means that the mode stores electric energy. This could be significantly helpful to optimally model the concerned structure.

In this paper, a $1 \times 2 \mathrm{MPA}$ array is proposed for wireless communications and analyzed using the source-free CMA which provides an insightful physical understanding of the operating mechanisms of the antenna. A complementary hexagonal split ring resonator (CHSRR) metamaterial is used for bandwidth enhancement of the $1 \times 2$ patch antenna array. Simulation and optimization of the proposed antenna structure are performed using the 3-D full-wave CST Microwave Studio software.

The remainder of the paper is organized as follows: Section 2 describes how the proposed antenna is designed. The analysis and the behavior of CHSRR as well as a discussion on the method developed for mutual coupling reduction using TCM are described in Sec. 3. The concept introduced is then verified by experiment in Sec. 4, which is followed by the conclusion in Sec. 5 .

\section{Antenna Design and MTM Unit- Cell Analysis}

The configurations of the single element, $1 \times 2$ element patch antenna and HSRR MTM are presented in this section. The scattering parameters of the HSRR extracted using the effective medium theory are also discussed.

\subsection{Single Element MPA}

Figure 1(a) shows a conventional single element MPA used here as a reference antenna with which to compare the attributes of the final proposed antenna. The antenna is designed using FR4 material with a thickness of $h=1.575 \mathrm{~mm}$, relative permittivity $\varepsilon_{\mathrm{r}}=4.3$, and loss tangent of 0.025 . As determined utilizing the transmission line model equations [21], the width and length of patch antenna are $9.2 \mathrm{~mm}$ and $6.5 \mathrm{~mm}$, respectively. The single element MPA is matched to a transmission line of $50 \Omega$ characteristics impedance using a quarter-wave impedance transformer. The return loss of the antenna is minimized to reach $-25 \mathrm{~dB}$ at $10 \mathrm{GHz}$, as depicted in Fig. 1(b). The corresponding gain and bandwidth of this reference antenna are determined to be $5.36 \mathrm{~dB}$ and $620 \mathrm{MHz}$, respectively.

The main objective here is to enhance the performance of this reference antenna so that it can be utilized for 


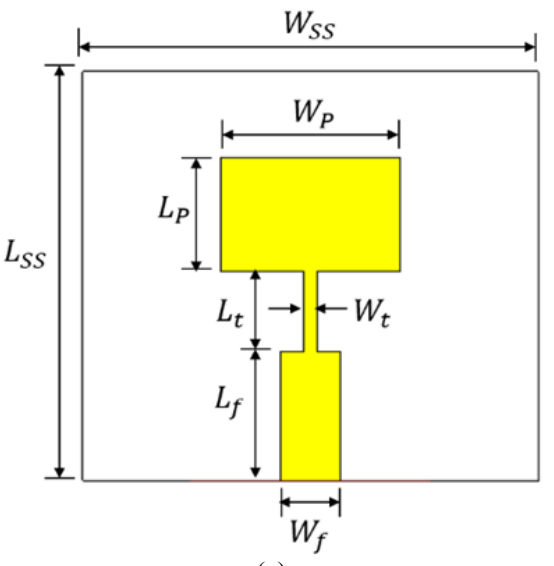

(a)

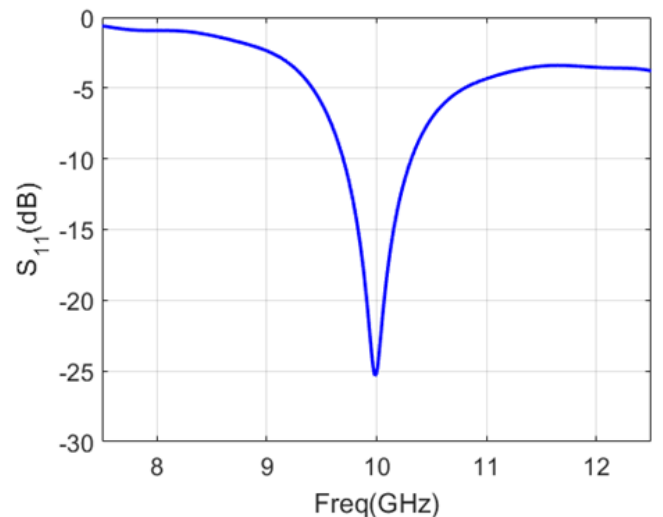

(b)

Fig. 1. Reference single element MPA operating at $10 \mathrm{GHz}$ : (a) Top view, (b) $S_{11}$ return loss.

MTM-based wireless communications using TCM. Enhanced antenna performance refers to an improvement to the gain, bandwidth, directivity, and other functions which are difficult to achieve with a single element patch. In the following sub-sections, the single element antenna is initially transformed to a $1 \times 2$ array, following which; five HSRRs are etched within the ground plane to widen the antenna bandwidth. Subsequently, a superstrate MTM layer covers the patch to magnify the antenna gain significantly.

\section{2. $1 \times 2$ Element MPA Array}

Figure 2(a) shows the $1 \times 2$ MPA array. The two patches have the same dimensions of the single patch antenna referred to above, and are constructed on the same kind of substrate. The overall dimensions of the substrate are $35 \times 28 \mathrm{~mm}^{2}$. The spacing between the rectangular patch array elements, about $9 \mathrm{~mm}$, is slightly greater than $\lambda_{\mathrm{g}} / 2$, where $\lambda_{\mathrm{g}}$ is the guided wavelength at $10 \mathrm{GHz}$. As determined utilizing the equations presented in [28], the input impedance at the edge of the rectangular patch is $225 \Omega$ at resonance. A corporate feeding network is used for equal division of power. Three different widths of $50 \Omega$, $70.7 \Omega$ and $100 \Omega$ microstrip lines are used for the feeding network. As determined from mathematical equations reported in [29], the widths of the quarter wavelength transformer $106 \Omega, 50 \Omega, 70.7 \Omega$ and $100 \Omega$ microstrip transmission lines are $0.6,3.6,2.1$ and $0.71 \mathrm{~mm}$, respectively. The

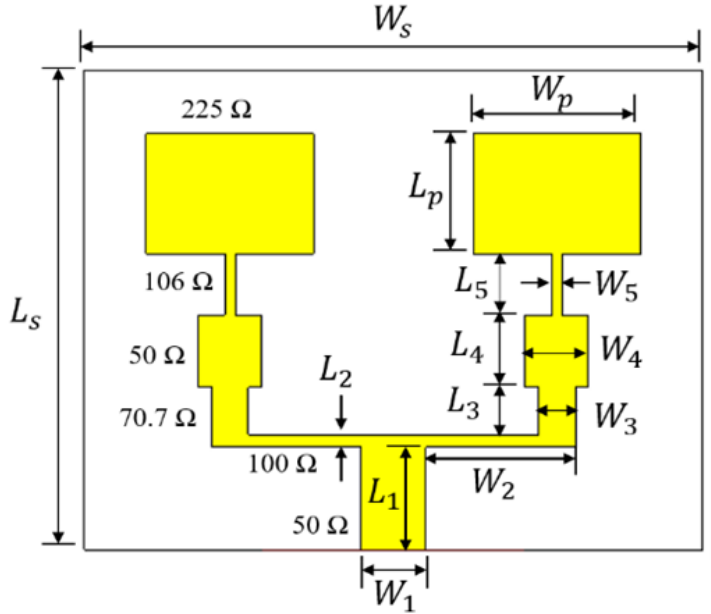

(a)

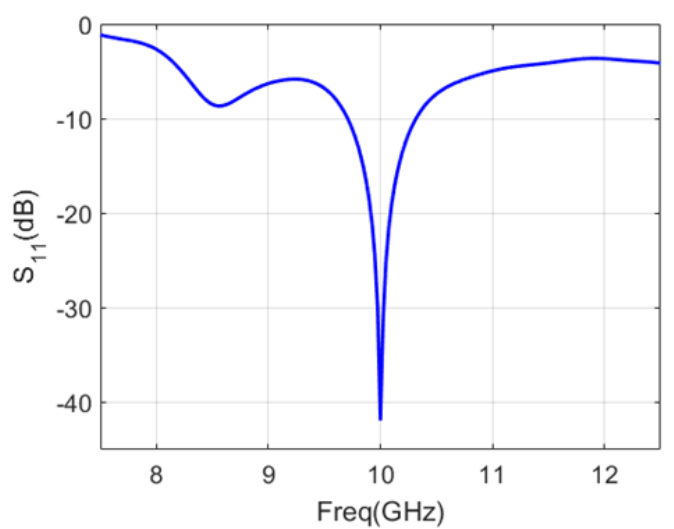

(b)

Fig. 2. 2-element patch antenna array operating at $10 \mathrm{GHz}$ : (a) schematic diagram, (b) $\mathrm{S}_{11}$ return loss.

lengths of these lines are $3.6,6,3.5$ and $4.35 \mathrm{~mm}$ in the same sequences. The return loss of the $1 \times 2$ rectangular microstrip patch array is optimized to attain $-41 \mathrm{~dB}$ at $10 \mathrm{GHz}$ as shown in Fig. 2(b). The corresponding antenna gain and bandwidth are accomplished to $8.25 \mathrm{~dB}$ and $610 \mathrm{MHz}$.

\subsection{HSRR Unit Cell Design and Extraction of Its Effective Parameters}

The HSRR structure consists of two nested concentric metallic hexagonal rings with a split in the opposite sides of each as illustrated in Fig. 3(a). This single HSRR unit cell is modeled on the CST microwave studio software where the boundary conditions are set as shown in Fig. 3(b). The S-parameters over the frequency range from 7 to $13 \mathrm{GHz}$ are presented in Fig. 3(c). It can be observed that the unit cell resonates at $10 \mathrm{GHz}$ with a return loss of $-16 \mathrm{~dB}$. It acts as an electrically small $\mathrm{LC}$ resonator with distributed capacitance and inductance. Based on Babinet's principle and the duality concept, the CSRR is the negative image of SRR, but the basic mechanism is the same for both resonators.

Figure 3(d) shows the negative permittivity characteristics of the CHSRR which are extracted from the scattering parameters using the algorithm presented in [30]. 


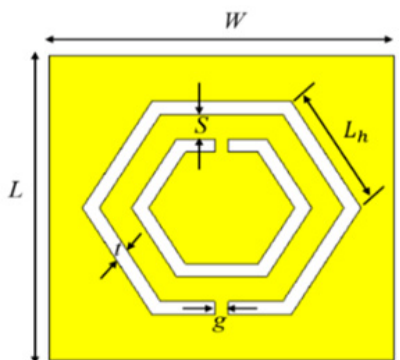

(a)

(c)

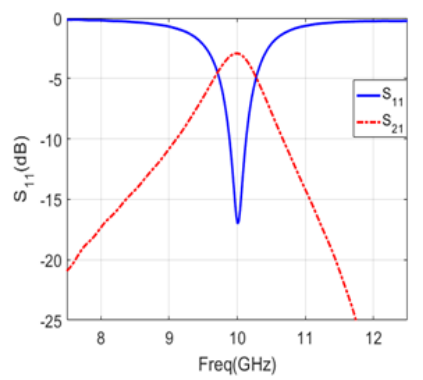

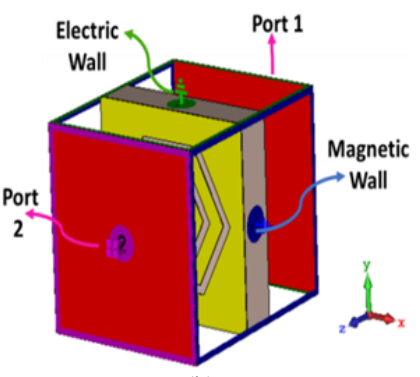

(b)

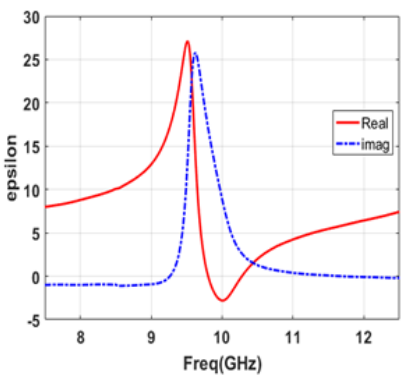

(d)
Fig. 3. CHSRRs: (a) Schematic diagram, (b) modeling setup and boundary conditions, (c) S-parameters, (d) extracted permittivity.

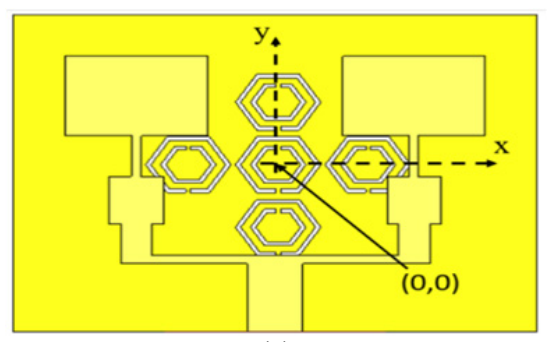

(a)

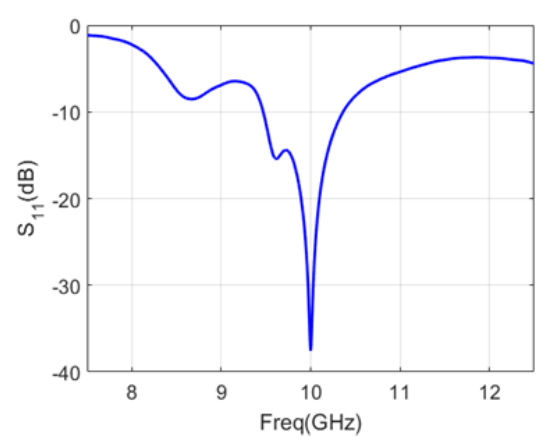

(b)

Fig. 4. Proposed antenna incorporating 5 HCSRR MTM unit cells in the ground plane: (a) Schematic diagram and (b) S11 parameters.

\begin{tabular}{|c|c|c|c|c|c|c|}
\hline Parameter & $W_{\mathrm{s}}$ & $L_{\mathrm{s}}$ & $W_{\mathrm{p}}$ & $L_{\mathrm{p}}$ & $W$ & $L$ \\
\hline Value & 35 & 28 & 9.2 & 6.5 & 6 & 6 \\
\hline Parameter & $W_{1}$ & $L_{1}$ & $W_{2}$ & $L_{2}$ & $W_{3}$ & $L_{3}$ \\
\hline Value & 3.6 & 6 & 4.35 & 0.71 & 2.1 & 3.5 \\
\hline Parameter & $W_{4}$ & $L_{4}$ & $W_{5}$ & $L_{5}$ & $S$ & $L_{\mathrm{h}}$ \\
\hline Value & 3.6 & 4.2 & 0.6 & 3.6 & 0.5 & 1.41 \\
\hline Parameter & $W_{\mathrm{f}}$ & $L_{\mathrm{f}}$ & $t$ & $W_{\mathrm{t}}$ & $L_{\mathrm{t}}$ & $g$ \\
\hline Value & 3.1 & 6.9 & 0.3 & 0.74 & 4.3 & 0.28 \\
\hline
\end{tabular}

Tab. 1. Optimal values of the proposed antenna dimensions $(\mathrm{mm})$.
In order to enhance the bandwidth of the proposed antenna that resonates at $10 \mathrm{GHz}$, five hexagonal CSRRs are etched on the ground plane as shown in Fig. 4(a). It is evident in Fig. 4(b) that the band width is enlarged significantly up to $906 \mathrm{MHz}$. All optimal dimensions of the antenna and the HSRR unit cell are listed in Tab. 1 as done in [31].

\section{CMA of Proposed Antenna Elements and CHSRR Unit Cell}

The characteristic mode analysis (CMA) of the proposed antenna is carried out using the commercial simulator software CST MWS (ver. 2018) with a multilayer solver. The theory of Characteristic Mode Analysis is performed here in a series of steps to investigate how each part of the antenna structure influences the whole antenna performance. First, we start by examining the ground plane only by TCM. We then analyze the $1 \times 2$ MPA array including the ground plane but without MTM unit cells. Finally, we investigate the $1 \times 2$ MPA array with the five CHSRRs MTM unit cells inserted in the ground plane. These three analytical steps are performed without involving the feeding port. The main objective here is to understand how mutual coupling between the patch elements can be controlled using the MTMs by studying the behavior of the CMs. First, the modes are identified in the frequency band of interest and then the CHSRRs are implemented in the ground plane to block the modes that produce high coupling. The characteristic angles for the first four modes from the TCM analysis of the ground plane are demonstrated in Fig. 5, while the current distributions for the same modes are depicted in Fig. 6.

From a physical point of view, a characteristic angle models the phase angle between the characteristic current $J_{n}$ and the associated characteristic field $E_{n}$. Figure 5 presents the variation of the characteristic angles $\beta_{n}$ associated with the current modes versus frequency. It can be seen that a mode resonates when its characteristic angle $\beta_{n}$ is $180^{\circ}$. Therefore, when the characteristic angle is close to $180^{\circ}$ the mode is a good radiator mode; otherwise the mode mainly stores energy. In the case of $90^{\circ}<\beta_{n}<180^{\circ}$, the associated modes are inductive modes while in the case of $180^{\circ}<\beta_{n}<270^{\circ}$, the associated modes are capacitive modes. It is observed in Fig. 5 that only Modes 1 and 2 are contributing in the band of interest, while Modes 3 and 4 start contributing after $4.1 \mathrm{GHz}$ and after $14 \mathrm{GHz}$, respectively.

It is also observed in Fig. 6 that the current distribution of Mode 1 is concentrated around the sides of the ground while there is nearly no current (nulls) in the medial area of the ground that represents the important region between the two patches that determines whether the mode is one of coupling or non-coupling. Accordingly, Mode 1 is the non-coupling mode while Mode 2 represents the coupling mode because there is maximal current at the center region. We would want to block this mode that is causing high coupling. 


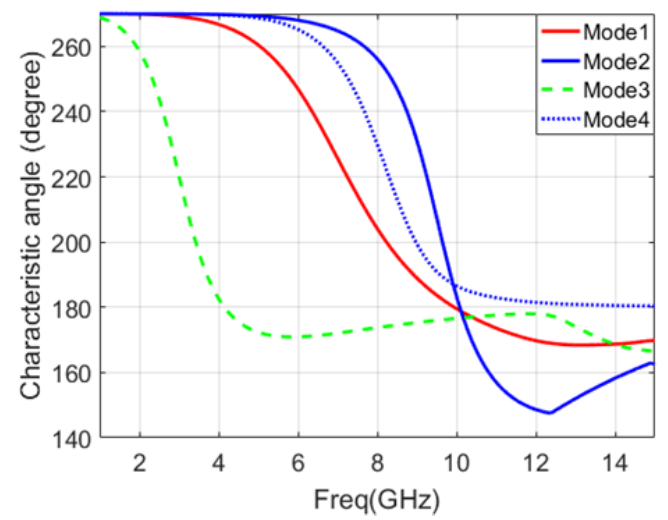

Fig. 5. Characteristic angle curves of the ground plane.

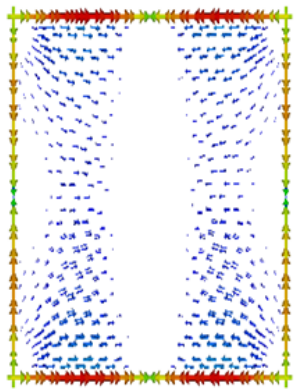

(a)

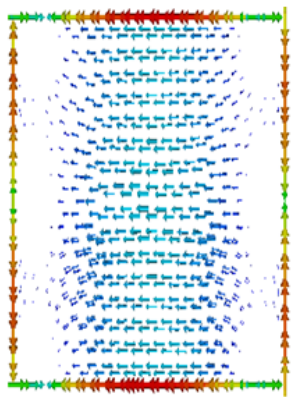

(c)
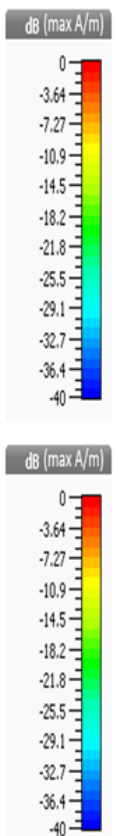

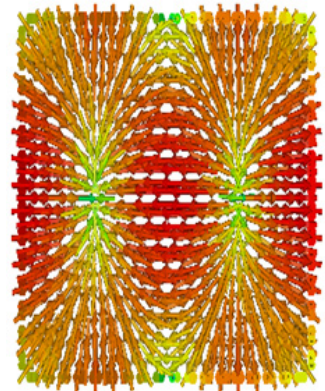

(b)

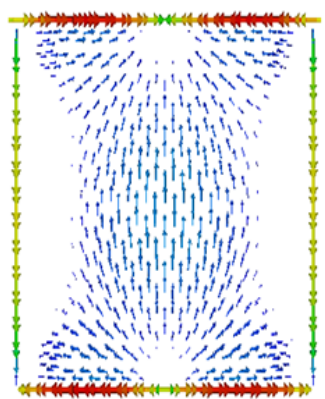

(d)
Fig. 6. Current distribution of the first four modes on the ground plane at $10 \mathrm{GHz}$ : (a) Mode 1, (b) Mode 2, (c) Mode 3, (d) Mode 4.

\section{1 $1 \times 2$ Element MPA Array without CHSRR MTM}

TCM is applied here to the $1 \times 2$ array patch antenna design including its substrate as in Fig. 2(a). The characteristic angle curves and the modal current distributions on the surface of the ground plane at $10 \mathrm{GHz}$ are shown in Figs. 7 and 8, respectively. It is clearly seen in Figs. 7 and 8 that it is not just the ground that decides the CM behavior. Modes 1 and 2 are contributing in the desired frequency band, while Mode 3 and Mode 4 start contributing only after $6.4 \mathrm{GHz}$ and $12.5 \mathrm{GHz}$, respectively. By using the current distribution, we can identify any of these desired frequency modes (Mode 1 and Mode 2) which cause high coupling between the two elements of the MPA array that we are going to block. It is also observed from Fig. 8 that Mode 2 causes high coupling between radiating elements because of the high current density in the area between the radiating elements.

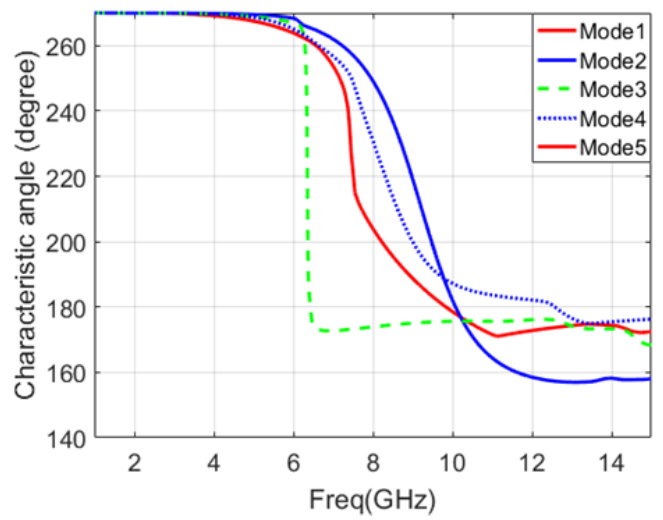

Fig. 7. Characteristics angle curves of the $1 \times 2$ array patch antenna in the presence of the FR4 substrate.

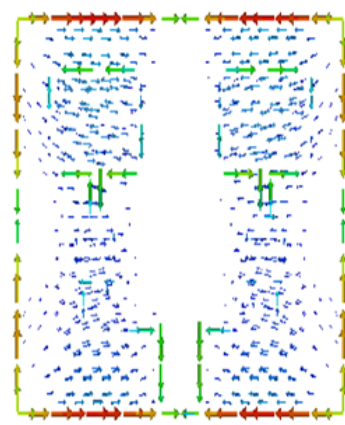

(a)

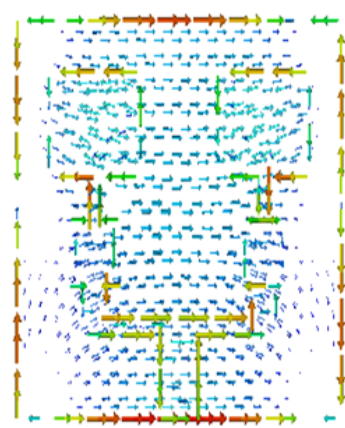

(c)
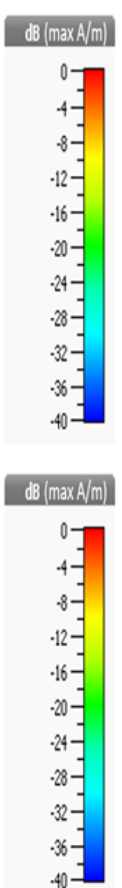

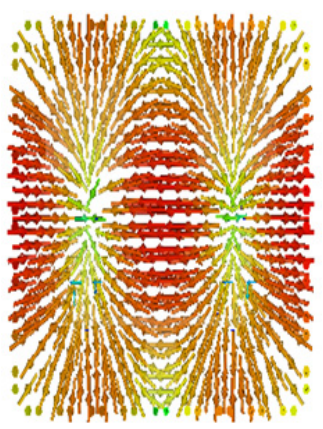

(b)

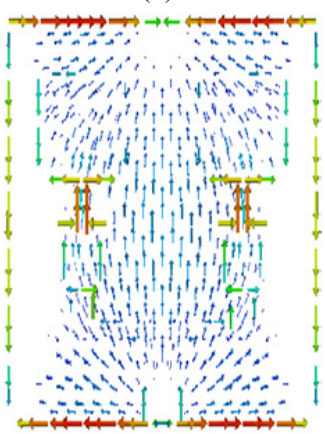

(d)
Fig. 8. Current distribution of the first four modes of the $1 \times 2$ patch array at $10 \mathrm{GHz}$ : (a) Mode 1, (b) Mode 2, (c) Mode 3, (d) Mode 4.

\section{2 $1 \times 2$ Element MPA Array with CHSRRs MTM}

The $1 \times 2$ element patch array incorporating the CHSRR MTM unit cells is analyzed here using TCM. As mentioned previously (see Sec. 3.1 above), Figure 7 shows that Modes 1 and 2 are contributing to the radiating bandwidth in the band of interest while Modes 3 and 4 are not contributing in any way. Moreover, in view of the current directions, it can be seen that Mode 1 is not contributing to the coupling between the two patch elements due to the presence of current nulls while Mode 2 produces coupling between them. To reduce the coupling between the two radiating elements, five hexagonal $\mathrm{CSRR}_{\mathrm{S}}$ are etched in the ground plane as shown in Fig. 4(a). Based on the characteristic mode analysis, the hexagonal $\mathrm{CSRR}_{\mathrm{S}}$ ought to be placed at a position such that they are able to block the 
coupling modes, without influencing or prohibiting the non-coupling modes. In the event that there are two modes in the coveted band where one is a coupling mode and the other a non-coupling mode, they can be isolated in such a way where one can be hindered without influencing the other. It gets more complicated whenever there is need to deal with additional modes. The characteristic angle curves and the $\mathrm{CM}$ current distribution at $10 \mathrm{GHz}$ are shown in Figs. 9 and 10, respectively. From these curves, it can be seen that just Mode 1 is available in the desired bandwidth area while Mode 2 is hindered in the band of interest. We can infer that Mode 1 is responsible for the radiation. The radiating $\mathrm{BW}$ of Mode 1 and the impedance $\mathrm{BW}$ of the actual structure stops exactly at a similar point, as shown in Fig. 9. This implies Mode 1 is assuming the significant role of the desired resonance frequency.

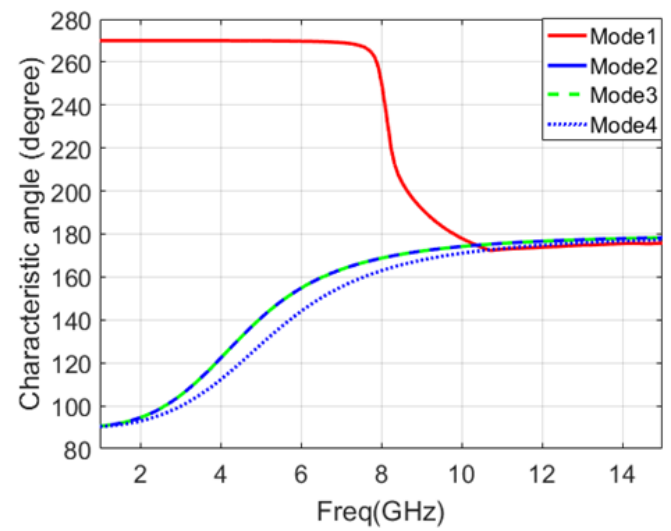

Fig. 9. Characteristic angle curves of the $1 \times 2$ array patch with CHSRRS.

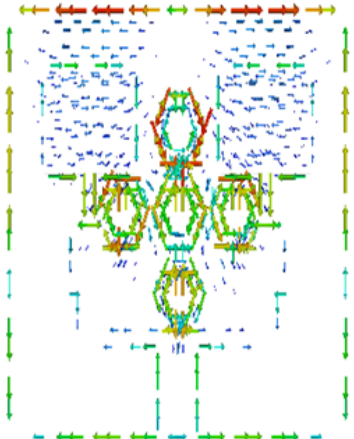

(a)

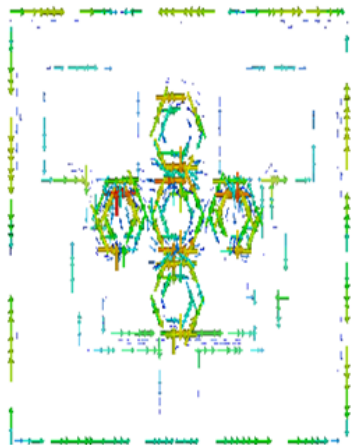

(c)

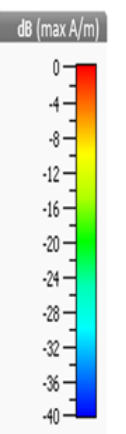

1

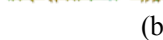

(b)

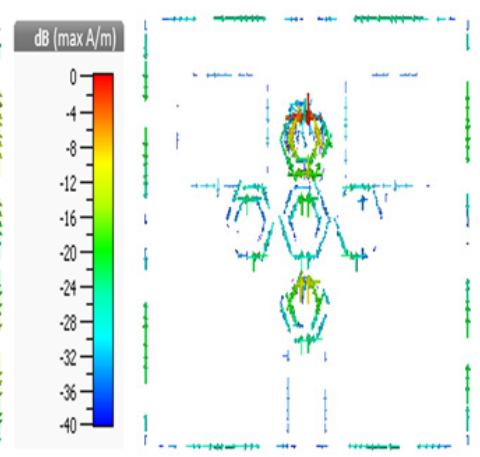

(d)
Fig. 10. Current distributions of the first four modes of the $1 \times 2$ array patch at $10 \mathrm{GHz}$, where (a) Mode 1, (b) Mode 2, (c) Mode 3, (d) Mode 4.

\section{Measurements and Discussions}

The proposed $1 \times 2$ MPA array based on MTM was fabricated and measured using the R\&S ZVA 67 VNA (Vector Network Analyzer) operating from $10 \mathrm{MHz}$ to $67 \mathrm{GHz}$. Photographs of the fabricated prototype are shown in Fig. 11. Figure 12(a) depicts the simulated return loss characteristics of the reference antenna, $1 \times 2$ array MPA without (unloaded) and with (loaded) HSRR MTM unit cells on the ground plane, whereas Figure 12(b) displays the simulated and measured $S_{11}$ parameters of the proposed antenna under loaded conditions.

It can be observed from Fig. 12(a) that the array antenna without MTM resonates at $10 \mathrm{GHz}$ and has a bandwidth of $610 \mathrm{MHz}$, whereas the bandwidth expands to $906 \mathrm{MHz}$ when five HSRRs MTM unit cells are incorporated
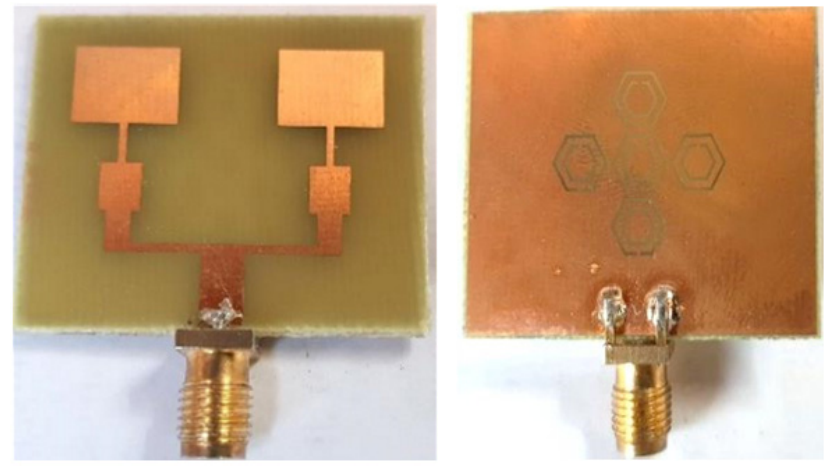

Fig. 11. Fabricated model: (a) top and (b) bottom views.

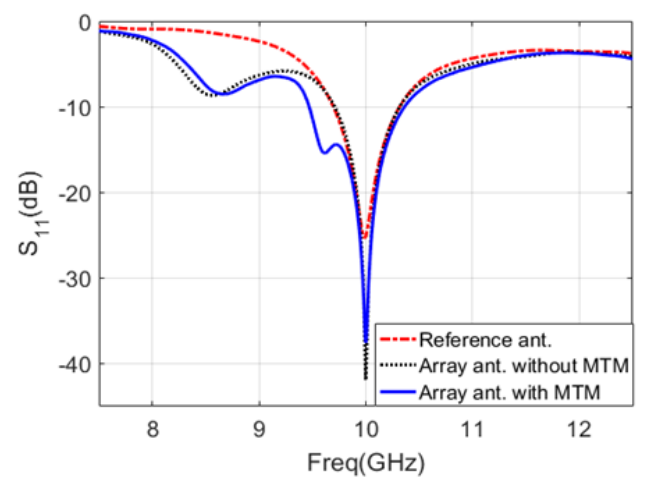

(a)

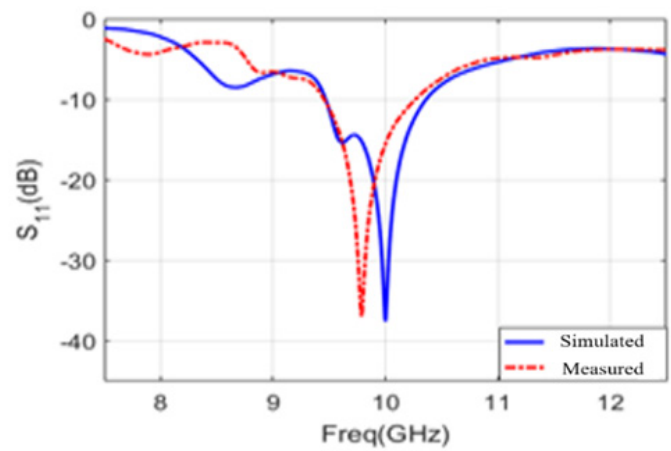

(b)

Fig. 12. Return loss characteristics of (a) Reference antenna and array antenna without and with MTM (simulated). (b) Proposed array antenna with MTM (measured and simulated). 
on the ground plane, Figure 12(b) demonstrates the simulation and measurement results which are in good agreement with each other. However, there is a small shift in the resonant frequency between simulated and measured return loss (about $2 \%$ ) due to fabrication tolerance.

The SatimoStar Lab near-field antenna measurement system setup with measurement ranges of $800 \mathrm{MHz}$ to $6 \mathrm{GHz}$, and $6 \mathrm{GHz}$ to $18 \mathrm{GHz}$ is shown in Fig. 13. It is used to measure the antenna gain, radiation efficiency and radiation pattern characteristics. This system allows measuring the antenna's electric fields within the near-field region to compute the corresponding far-field values of the antenna under test (AUT). The AUT is placed on the test board and positioned in the middle of a circular "arch" which contains 29 measuring probe antennas divided into 15 probes for low frequency range and the other 14 probes for high frequency range. These probes are placed at equal distance surrounding the circular surface. The AUT is rotated horizontally over $360^{\circ}$, and this rotation and array of probes together perform a full 3D scan of the AUT and collect data for radiation patterns. The far-field data is then used to compute the gain and radiation efficiency of the AUT.

Figure 14 compares the simulated and measured radiation patterns in the two principal planes. It is observed here that simulated patterns matched well with the measurements at the resonant frequency.

The antenna broadband gain and radiation efficiency are also measured and displayed in Fig. 15 and Fig. 16, respectively. It is clear from Fig. 15 that the simulated gain is enriched from $5.38 \mathrm{~dB}$ to $8.25 \mathrm{~dB}$ when the single element antenna is transformed to a $1 \times 2$ array and a good agreement between simulated and measured gain is achieved.
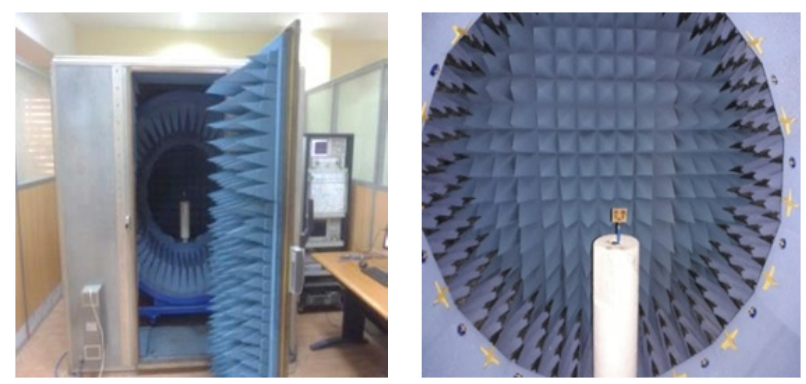

Fig. 13. Measurement setup in the SatimoStar Lab.

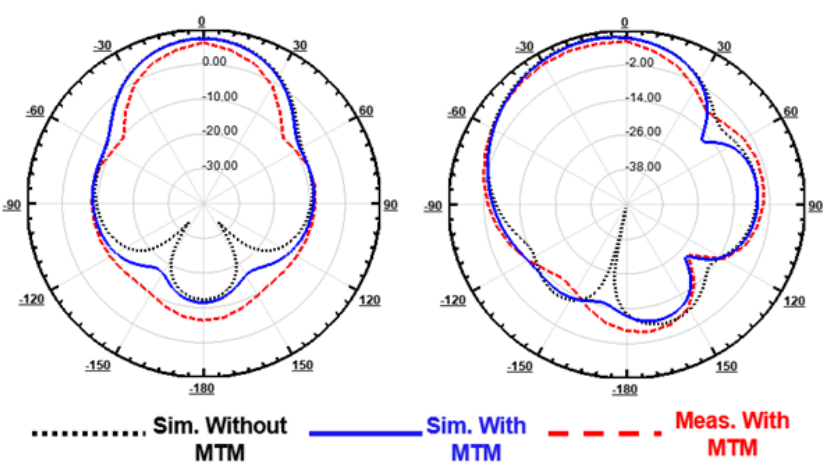

Fig. 14. Simulated and measured radiation patterns at $10 \mathrm{GHz}$ : (a) E-plane, (b) H-plane.

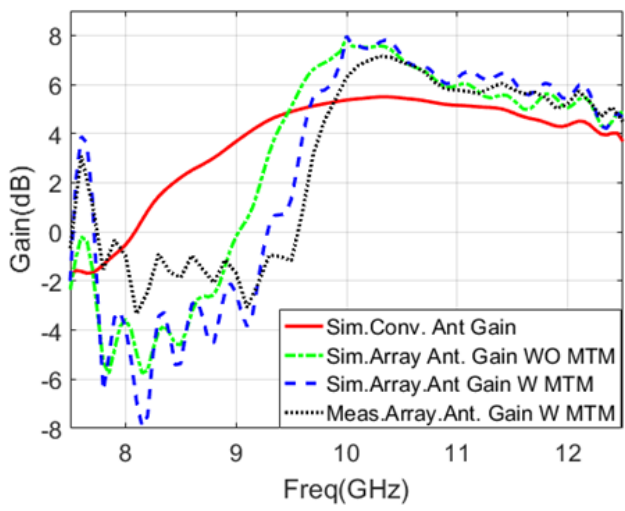

Fig. 15. Comparison between the measured and simulated gains of the proposed antennas with and without HSRR MTM.

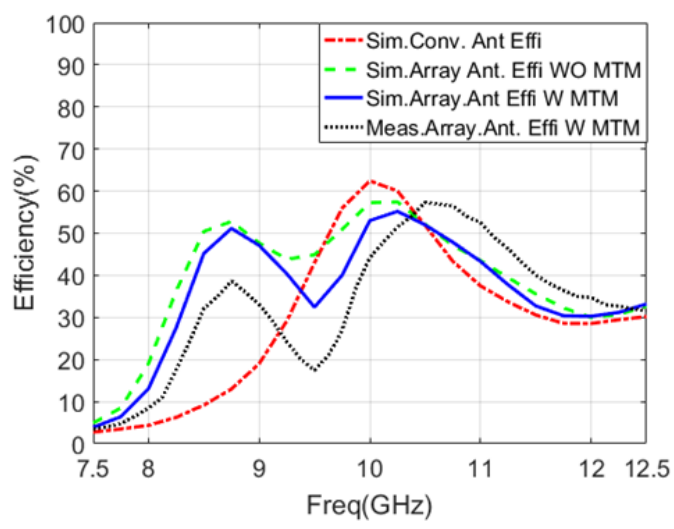

Fig. 16. Comparison between measured and simulated total efficiencies of the proposed antenna with and without HSRR MTM.

The ratio of the radiated power by the antenna to the power received by the antenna terminal is defined as the antenna efficiency. As shown in Fig. 16, the efficiency of the conventional single patch antenna is greater than the efficiency of the 2-element patch antenna, because the greater the size of substrate the more the surface wave and thus reduces the efficiency. In Fig. 16, the measured and simulated total efficiencies of the proposed antenna are roughly matching within the antenna band of interest.

Comparisons between the results achieved in this research with available latest literature are reported in Tab. 2 . Most of the antennas have good results, while our proposed antenna uses a novel method of exciting different characteristic modes to obtain better coupling reduction.

\section{Conclusions}

A two-element MPA array with five HSRR MTM unit cells etched on the ground plane is proposed for wireless communications. CMA has been shown to be an effective approach to modeling and designing the proposed antenna. The mode contributing to coupling was identified and blocked by introducing the HSRR MTM unit cells at the position of that mode. The advantage of HSRRs is that they block the mode that produces coupling without affecting 


\begin{tabular}{|c|c|c|c|c|c|c|}
\hline Reference & $\begin{array}{c}\text { Dimensions } \\
\left(\mathrm{mm}^{2}\right)\end{array}$ & Decoupling technique & $\begin{array}{c}\text { Max. isolation } \\
\text { improvement }(\mathrm{dB})\end{array}$ & Bandwidth (MHz) & Gain (dB) & Efficiency (\%) \\
\hline$[32]$ & $30 \times 30$ & DGS (U-shaped slots) & 10 & Not reported & Not reported & Not reported \\
\hline$[33]$ & $42 \times 10.2$ & MTM (Modified SRR) & Not reported & 115 & Not reported & 90 \\
\hline$[34]$ & $36 \times 36$ & Metalized walls and strips & 13 & $\begin{array}{c}58 \text { and } 43 \\
(\text { dual band) }\end{array}$ & $\begin{array}{c}2.7 \text { and } 2.85 \\
\text { (dual band) }\end{array}$ & $\begin{array}{c}65 \text { and } 63 \\
(\text { dual band) }\end{array}$ \\
\hline$[35]$ & $150 \times 150$ & EBG & 20 & 85 & 7.1 & 90 \\
\hline$[36]$ & Not reported & Split-ring resonators & 13.54 & Not reported & 7.01 & Not reported \\
\hline This work & $35 \times 28$ & MTM and TCM & Not reported & 906 & 8.2 & 58 \\
\hline
\end{tabular}

Tab. 2. Comparison among the results obtained in this work and recent papers.

the other modes significantly. Loading of HSRRs does not only reject the higher harmonics, but also reduces the surface waves and mutual coupling between the elements of the patch antenna array. The proposed antenna enriches the gain of the conventional patch antenna by about $50 \%$ and also extends its bandwidth by about $50 \%$. The performance comparison of the antenna array with and without HSRR MTM provides verification that MTMs have good potential to improve significantly the performance of the MPA. TCM is useful and efficient in investigating and analyzing the performance of the proposed antenna. A prototype of the proposed antenna that was fabricated showed good agreement between measurement and simulation results.

\section{References}

[1] CHEN, S., ZHAO, J. The requirements, challenges, and technologies for $5 \mathrm{G}$ of terrestrial telecommunication. IEEE Communication Magazine, 2014, vol. 52, no. 5, p. 36-43. DOI: 10.1109/MCOM.2014.6815891

[2] HAMAD, E. K. I., ABDELAZIZ, A. Metamaterial superstrate microstrip patch antenna for $5 \mathrm{G}$ wireless communication based on the theory of characteristic modes. Journal of Electrical Engineering, 2019, vol. 70, no. 3, p. 187-197. DOI: $10.2478 /$ jee2019-0027

[3] FANG, D., QIAN, Y., HU, R. Q. Security for 5G mobile wireless networks. IEEE Access, 2018, vol. 6, p. 4850-4874. DOI: 10.1109/ACCESS.2017.2779146

[4] ABDELAZIZ, A., HAMAD, E. K. I. Design of a compact high gain microstrip patch antenna for tri-band $5 \mathrm{G}$ wireless communication. Frequenz, 2019, vol. 73, no. 1-2, p. 45-52. DOI: 10.1515/freq-2018-0058

[5] KUMAR, A., GUPTA, N., GAUTAM, P. C. Gain and bandwidth enhancement techniques in microstrip patch antennas $-\mathrm{a}$ review. International Journal of Computer Applications, 2016, vol. 148, no. 7, p. 9-14. DOI: 10.5120/ijca2016911207

[6] ISLAM, M. A., KARMAKAR, N. C. A 4×4 dual polarized mmwave ACMPA array for a universal mm-wave chipless RFID tag reader. IEEE Transactions on Antennas and Propagation, 2015, vol. 63, no. 4, p. 1633-1640. DOI: 10.1109/TAP.2015.2398355

[7] KOVITZ, J. M., SAMII, Y. R. Using thick substrates and capacitive probe compensation to enhance the bandwidth of traditional CP patch antennas. IEEE Transactions on Antennas and Propagation, 2014, vol. 62, no. 10, p. 4970-4979. DOI: 10.1109/TAP.2014.2343239
[8] ROW, J. S. A simple impedance-matching technique for patch antennas fed by coplanar microstrip line. IEEE Transactions on Antennas and Propagation, 2005, vol. 53, no. 10, p. 3389-3391. DOI: 10.1109/TAP.2005.856375

[9] FUKUSAKO, T., NAKANO, T. A compact patch antenna using artificial ground structure with high permittivity substrate. In IEEE-APS Topical Conference on Antennas and Propagation in Wireless Communications (APWC). Turin (Italy), 7-11 Sept. 2015, p. 1548-1549. DOI: 10.1109/APWC.2015.7300219

[10] MAHDI HONARI, M., ABDIPOUR, A., MORADI, G. Bandwidth and gain enhancement of an aperture antenna with modified ring patch. IEEE Antennas and Wireless Propagation Letters, 2011, vol. 10, p. 1413-1416. DOI: 10.1109/LAWP.2011.2178998

[11] ZHANG, C., CAO, X, GAO, J., et al. Wideband high-gain and low scattering antenna using shared-aperture metamaterial superstrate. Radioengineering, 2018, vol. 27, no. 2, p. 379-385. DOI: $10.13164 /$ re.2018.0379

[12] ALIBAKHShIKENARI, M., VIRDEE, B. S., ALI, A., et al. Miniaturised planar-patch antenna based on metamaterial L-shaped unit-cells for broadband portable microwave devices and multiband wireless communication systems. IET Microwaves, Antennas and Propagation, 2018, vol. 12, no. 7, p. 1080-1086. DOI: $10.1049 /$ iet-map.2016.1141

[13] VESELAGO, V. G. The electrodynamics of substances with simultaneously negative values of $\varepsilon$ and $\mu$. Soviet Physics Uspekhi, 1968, vol. 10, no. 4, p. 509-514. DOI: 10.1070/PU1968v010n04ABEH003699

[14] SMITH, D. R., PADILlA, W. J., VIER, D. C., et al. Composite medium with simultaneously negative permeability and permittivity. Physical Review Letters, 2000, vol. 84, no. 18 p. 4184-4187. DOI: $10.1103 /$ PhysRevLett.84.4184

[15] HAMAD, E. K. I., HAMDALLA, M. Z. M. Design of a compact dual-band microstrip antenna enabled by complementary split ring resonators for x-band applications. Advanced Electromagnetics, 2018, vol. 7, no. 3, p. 82-86. DOI: 10.7716/aem.v7i3.766

[16] SARAVANAN, M., GEO, V. B., UMARANI, S. M. Gain enhancement of patch antenna integrated with metamaterial inspired superstrate. Journal of Electrical Systems and Information Technology, 2018, vol. 5, no. 3, p. 263-270. DOI: 10.1016/j.jesit.2018.04.002

[17] KHANDElWAL, M. K., ARORA, A., KUMAR, S. Dual band double negative (DNG) metamaterial with small frequency ratio. Journal of Electromagnetic Waves and Applications, 2018, vol. 32, no. 17 , p. 2167-2181. DOI: 10.1080/09205071.2018.1498026

[18] ALI, W. A., HAMAD, E. K. I., BASSIUNY, M. A., HAMDALLAH, M. Z. Complementary split ring resonator based triple band microstrip antenna for WLAN/WIMAX applications. Radioengineering, 2017, vol. 26, no. 1, p. 78-84. DOI: $10.13164 /$ re.2017.0078 
[19] ELSHEAKH, D., ABDALLAH, E. Ultra-wide-bandwidth (UWB) microstrip monopole antenna using split ring resonator (SRR) structure. International Journal of Microwave and Wireless Technologies, 2018, vol. 10, no. 1, p. 123-132. DOI: $10.1017 / \mathrm{S} 1759078717001131$

[20] IBRAHIM, M. I., SAFWAT, A. M. E., EL-HENNAWY, H Single/dual-band CSRR-loaded differential-fed square patch antenna with monopolar radiation pattern. In The 33rd National Radio Science Conference (NRSC'16). Aswan (Egypt), 2016, p. 81-86. DOI: $10.1109 /$ NRSC.2016.7450827

[21] BALANIS, C. A. Antenna Theory: Analysis and Design. 4th ed. Hoboken (New Jersey): John Wiley \& Sons, Inc., 2016. ISBN: 978-1-118-64206-1

[22] DIALlO, A., LUXEY, C., LE THUC, P., et al. Enhanced twoantenna structures for universal mobile telecommunications system diversity terminals. IET Microwaves, Antennas and Propagation, 2008, vol. 2, no. 1, p. 93-101. DOI: 10.1049/ietmap:20060220

[23] ILUZ, Z., SHAVIT, R., BAUER, R. Microstrip antenna phased array with electromagnetic band gap substrate. IEEE Transactions on Antennas and Propagation, 2004, vol. 52, no. 6, p. 1446-1453. DOI: $10.1109 /$ TAP.2004.830252

[24] HAMAD, E. K. I., HAMDALlA, M, Z. M. Design of miniaturized and high isolation metamaterial-based MIMO antenna for mobile terminals. Journal of Engineering Sciences, Assiut University, 2017, vol. 45, no. 6, p. 764-773.

[25] GARBACZ, J. R., TURPIN, R. H. A generalized expansion for radiated and scattered fields. IEEE Transactions on Antennas and Propagation, 1971, vol. 19, no. 3, p. 348-358. DOI: 10.1109/TAP.1971.1139935

[26] HARRINGTON, R. F., MAUTZ, J. R. Theory of characteristic modes for conducting bodies. IEEE Transactions on Antennas and Propagation, 1971, vol. 19, no. 5, p. 622-628. DOI: 10.1109/TAP.1971.1139999

[27] CABEDO-FABRES, M., ANTONINO-DAVIU, E., VALERONOGUEIRA, A., et al. The theory of characteristic modes revisited: A contribution to the design of antennas for modern applications. IEEE Antennas and Propagation Magazine, 2007, vol. 49, no. 5, p. 52-68. DOI: 10.1109/MAP.2007.4395295

[28] GARG, R., BHARTIA, P., BHAL, I., ITTIPIBOON, A. Microstrip Antenna Design Handbook. Boston-London (UK): Artech House, 2001. ISBN: 978-0890065136

[29] POZAR, D. M. Microwave Engineering. New York (USA): John Wiley \& Sons, 2011. ISBN: 978-0470631553

[30] ZIOLKOWSKI, R. W. Design, fabrication, and testing of double negative metamaterials. IEEE Transactions on Antennas and Propagation, 2003, vol. 51, no. 7, p. 1516-1529. DOI: 10.1109/TAP.2003.813622

[31] BOSE, S., RAMRAJ, M., RAGHAVAN, S. Design, analysis and verification of hexagon split ring resonator based negative index metamaterial. In Annual IEEE India Conference (INDICON). Kochi (India), 2012. DOI: 10.1109/INDCON.2012.6420764

[32] KUN WEI, JIAN-YING LI, LING WANG, et al. Mutual coupling reduction of microstrip antenna array by periodic defected ground structures. In IEEE 5th Asia-Pacific Conference on Antennas and Propagation (APCAP 2016). Kaohsiung (Taiwan), 2016, p. 389-390. DOI: 10.1109/APCAP.2016.7843257

[33] KHARCHE, S., REDDY, G. S., MUKHERJEE, J., et al. Mutual coupling reduction by using tilted variable length SRR like structure in UWB MIMO antennas. In IEEE International Symposium on Antennas and Propagation \& USNC/URSI National
Radio Science Meeting. San Diego (CA, USA), 2017, p. 2285-2286. DOI: 10.1109/APUSNCURSINRSM.2017.8073185

[34] BOUKARKAR, A., LIN, X. Q., JIANG, Y., et al. A miniaturized extremely close-spaced four-element dual-band MIMO antenna system with polarization and pattern diversity. IEEE Antennas and Wireless Propagation Letters, 2018, vol. 17, no. 1, p. 134-137. DOI: 10.1109/LAWP.2017.2777839

[35] ZHAI, G., CHEN, Z. N., QING, X. Mutual coupling reduction of a closely spaced four-element MIMO antenna system using discrete mushrooms. IEEE Transactions on Microwave Theory and Techniques, 2016, vol. 64, no. 10, p. 3060-3067. DOI: 10.1109/TMTT.2016.2604314

[36] MUKHERJEE, B., PARUI, S. K. DAS, S. Mutual coupling reduction of microstrip antenna arrays using rectangular split ringshaped defected ground structure. In International Conference on Communications, Devices and Intelligent Systems (CODIS 2012). Kolkata (India), 2012, p. 202-204. DOI: 10.1109/CODIS.2012.6422172

\section{About the Authors...}

Ehab K. I. HAMAD earned his bachelor's and master's degrees in Electrical Engineering from Assiut University, Egypt in 1994 and 1999, respectively. He received his Ph.D. degree in Electrical Engineering from Otto-von-Guericke University Magdeburg, Germany in 2006. From 1996 to 2001 he was a Teaching/Research Assistant at Aswan Faculty of Engineering, South Valley University. From July 2001 to December 2006, he was a Research Assistant at the Chair of Microwave and Communication Engineering, Otto-von-Guericke University Magdeburg, Germany. From July 2010 to April 2011, he joined the School of Computing and Engineering, University of Huddersfield, UK as a Post-doctoral Research Assistant. Dr. Hamad is currently an Associate Professor for microwave antennas at the Department of Electrical Engineering, Faculty of Engineering, Aswan University, Aswan, Egypt. Dr. Hamad has authored and co-authored over 40 technical peer reviewed papers in international journals and conference proceedings and he received 1 best paper award. His current research interests include the antenna design, MIMO antennas, $\mathrm{mm}$ wave antennas, multiband/wideband small antennas for $4 \mathrm{G} / 5 \mathrm{G}$, metamaterials, UWB, RFID, and characteristics mode analysis.

Ahmed ABDELAZIZ received the B.E. degree in Electronic and Communication Engineering from the Arab Academy for Science, Technology and Maritime Transport, Egypt in 2014. He earned his Master Degree in Electrical Engineering with thesis entitled "Design of Microstrip Antennas Using Metamaterials and Characteristics Mode Theory for 5G Wireless Communications" from the Faculty of Engineering, Aswan University in February 2019. Eng. Ahmed is working now as a Teaching/Research Assistant at the Higher Institute of Engineering and Technology, Luxor, Egypt. His current research interests include the antenna design, metamaterials, characteristic mode theory, and 5G. 